\title{
Apport de I'IRTF et de la rhéologie à l'étude de la stabilité d'émulsions cosmétiques H/E
}

\author{
H. Masmoudi ${ }^{\star a}$, L. Douifib, Y. Le Dréaua, P. Piccerelle ${ }^{\mathrm{b}}$ et J. Kister ${ }^{\mathrm{a}}$ \\ *houdamas@yahoo.fr Tel: +3349128 13 Fax: +33491636509
}

\begin{abstract}
aLaboratoire GOAE, CNRS UMR 6171, service 561, Université Paul Cézanne - Aix-Marseille III, Avenue Escadrille Normandie Niemen 13397 Marseille cedex 20, France.

bLaboratoire de Pharmacie Galénique, Faculté de Pharmacie, Université d'Aix-Marseille II, 27 bd. Jean Moulin 13385 Marseille cedex 05, France.
\end{abstract}

Résumé : Les réactions de vieillissement concernent différentes phases de la vie d'un produit : le stockage, la péremption du produit après ouverture et les problèmes environnementaux "d'après vie". Le but de ce travail a été de montrer l'intérêt de la Spectrométrie Infrarouge à Transformée de Fourier (IRTF) et de la rhéologie pour étudier la stabilité d'émulsions cosmétiques principalement au stockage. Dans cette étude, le stockage à long terme à $50^{\circ} \mathrm{C}$ pendant six mois est utilisé pour accélérer le vieillissement et évaluer la stabilité de quatre types d'émulsions $\mathrm{H} / \mathrm{E}$. L'évolution du vieillissement de ces crèmes a été suivie en IRTF par le biais d'indices spectrométriques et en rhéologie dynamique par la mesure des modules élastique et visqueux G' et G" ainsi que celle du seuil d'écoulement $\tau_{0}$ en fonction du temps. Ces études ont été complétées par des mesures en microscopie optique à contraste de phase.

Durant le processus de vieillissement, des modifications chimiques ont été mesurées par IRTF mettant en évidence l'apparition de nouvelles espèces issues de l'oxydation des émulsions. La déconvolution des spectres a permis d'identifier ces espèces et d'aborder les mécanismes d'oxydation des émulsions. En outre, les mesures rhéologiques réalisées en parallèle révèlent un aspect prédictif de cette technique puisque des modifications sont mesurées pour des émulsions stables à l'œil nu et pour lesquelles aucune modification chimique n'est mesurée par IRTF. Par conséquent, l'utilisation de ces deux techniques a permis, d'une part, de mettre en évidence l'influence de la nature de l'huile et de l'émulsifiant sur la stabilité des émulsions cosmétiques, et d'autre part, d'identifier les produits de dégradation issus du vieillissement et de faciliter le choix d'additifs.

Mots clés : émulsion cosmétique, stabilité au stockage, spectrométrie infrarouge, rhéologie, oxydation, vieillissement, microscopie en contraste de phase

\section{Introduction}

Les émulsions $\mathrm{H} / \mathrm{E}$ "huile dans eau" ou $\mathrm{E} / \mathrm{H}$, qui représentent la majeure partie des produits cosmétiques, sont thermodynamiquement instables: ces systèmes évoluent lentement vers la séparation des phases aqueuses et grasses. Les constituants d'une émulsion doivent donc se séparer à plus ou moins long terme en deux phases distinctes aboutissant donc à la destruction du produit cosmétique. L'instabilité des émulsions se manifeste plus ou moins rapidement par différents phénomènes de déstabilisation: le crémage, la floculation, la coalescence, le mûrissement d'Oswald... [1-4]. Connaître la durée de stabilité d'une crème (émulsion $H / E$ ou $E / H$ ) représente encore une des étapes les plus longues et les plus difficiles pour les formulateurs.

Ainsi, pouvoir déceler l'instabilité d'une émulsion avant qu'elle ne soit visible à l'œil nu serait un gain de temps considérable au niveau de la formulation. Compte tenu de la mise en place des nouvelles normes européennes (programme "REACH"), un des paramètres importants du contrôle qualité en formulation est de pouvoir suivre une cinétique de vieillissement en étant capable d'identifier les produits de dégradation pouvant être toxiques. 
Jusqu'à présent, la Spectrométrie Infrarouge à Transformée de Fourier (IRTF) a surtout été utilisée en cosmétologie pour suivre la dégradation des matières premières $[5,6]$ ou pour mesurer le degré d'hydratation de la peau lors de l'application des crèmes [7]. L'innovation apportée par ce travail a été d'utiliser cette technique pour mettre en évidence les mécanismes d'oxydation se produisant pendant les réactions de vieillissement des émulsions cosmétiques $H / E$. Ainsi, les formulateurs pourront déterminer rapidement quelles seront les émulsions les plus sensibles à l'oxydation et choisir les additifs les plus adaptés pour lutter contre ce phénomène de vieillissement.

Le premier objectif est d'identifier les modifications structurales au sein des émulsions engendrées par un stockage à long terme (six mois). Le second objectif est de comparer les résultats obtenus par IRTF, mettant en évidence les modifications chimiques des émulsions, avec ceux obtenus en rhéologie dynamique.

\section{Etude de stabilité (stockage à long terme)}

Une étude de stabilité a été réalisée sur quatre types d'émulsions $\mathrm{H} / \mathrm{E}$ pendant six mois. Pour tester leur stabilité et accélérer le vieillissement ces émulsions sont stockées à $25^{\circ} \mathrm{C}$ et $50^{\circ} \mathrm{C}$. La reproductibilité de l'étude a été évaluée en formulant quatre lots de chaque type d'émulsions.

\section{Préparation des émulsions}

Les quatre types d'émulsions H/E (NAE1, NAE2, OE1, OE2) de composition classique sont préparées selon un processus direct d'émulsification [8]. La composition de ces émulsions est donnée dans le tableau 1. Ces émulsions se différencient soit par la nature de l'huile, soit par la nature de l'émulsifiant dans le but d'étudier l'influence de ces composés sur la stabilité des émulsions.

Tableau 1 : Composition des émulsions formulées.

\begin{tabular}{|c|c|c|c|c|}
\hline & NAE1 & NAE2 & OE1 & OE2 \\
\hline $\begin{array}{l}\text { Huile } \\
20.0 \%\end{array}$ & $\begin{array}{l}\text { Noyaux } \\
\text { d'abricot }\end{array}$ & $\begin{array}{l}\text { Noyaux } \\
\text { d'abricot }\end{array}$ & Olive & Olive \\
\hline $\begin{array}{c}\text { Emulsifiant } \\
5.0 \%\end{array}$ & Montanov 68 & Montanov 82 & Montanov 68 & Montanov 82 \\
\hline $\begin{array}{c}\text { Humectant } \\
5.0 \%\end{array}$ & Glycérine & Glycérine & Glycérine & Glycérine \\
\hline $\begin{array}{c}\text { Conservateur } \\
0.3 \%\end{array}$ & $\begin{array}{c}\text { Methylparaben/ } \\
\text { Propylparaben } \\
(50: 50)\end{array}$ & $\begin{array}{c}\text { Methylparaben/ } \\
\text { Propylparaben } \\
(50: 50)\end{array}$ & $\begin{array}{c}\text { Methylparaben/ } \\
\text { Propylparaben } \\
(50: 50)\end{array}$ & $\begin{array}{c}\text { Methylparaben/ } \\
\text { Propylparaben } \\
(50: 50)\end{array}$ \\
\hline $\begin{array}{c}\text { Eau } \\
\text { Ad. } 100.0 \%\end{array}$ & $\begin{array}{c}\text { Eau } \\
\text { démineralisée }\end{array}$ & $\begin{array}{c}\text { Eau } \\
\text { démineralisée }\end{array}$ & $\begin{array}{c}\text { Eau } \\
\text { démineralisée }\end{array}$ & $\begin{array}{c}\text { Eau } \\
\text { démineralisée }\end{array}$ \\
\hline
\end{tabular}

L'évolution de ces crèmes a été suivie en IRTF par le biais d'indices spectrométriques et en rhéologie dynamique par la mesure des modules élastique et visqueux G' et G" ainsi que celle du seuil d'écoulement $\tau_{0}$ en fonction du temps. Ces études ont été complétées par des mesures en microscopie optique à contraste de phase.

\section{La spectrométrie IRTF}

L'avantage de la spectrométrie Infra Rouge à Transformée de Fourier (IRTF) est son caractère non destructif, sa sensibilité et sa facilité d'utilisation. Cette technique permet d'identifier les produits 
de dégradation qui apparaissent lors de l'oxydation. II a semblé intéressant d'utiliser cette technique pour suivre l'évolution de la stabilité de formulations telles que les émulsions cosmétiques H/E dans leur ensemble et non pas, comme cela se fait actuellement, uniquement celle des matières premières. En raison des interactions chimiques, les produits d'oxydation d'un mélange peuvent différer de ceux des matières premières individuelles. Ainsi, l'IRTF, en appréhendant les mécanismes d'oxydation qui se produisent pendant les phénomènes de vieillissement des émulsions cosmétiques, permettra d'améliorer les formulations. En effet, la connaissance de ces mécanismes permettra de choisir les composés les plus stables qui de surcroît ne donneront pas de produits de dégradation toxiques.

Pour quantifier les espèces chimiques provenant de l'oxydation des émulsions, un microscope Infra Rouge à Transformée de Fourier Nicolet Protégé 460 en mode transmission a été utilisé. Les spectres obtenus ont été enregistrés entre 4000 et $650 \mathrm{~cm}^{-1}$ avec 64 acquisitions afin d'augmenter le rapport signal sur bruit. L'émulsion est déposée en film mince sur une lame de ZnSe (13 mm de diamètre, 1 $\mathrm{mm}$ d'épaisseur). La faible épaisseur de produit déposé permet l'évaporation de l'eau contenue dans l'émulsion ce qui supprime les problèmes de saturation du spectre. A partir d'un même dépôt, il est possible d'enregistrer plusieurs spectres grâce à une platine motorisée. Pour assurer la répétabilité des résultats, 10 spectres ont été enregistrés pour chaque échantillon (deux dépôts et cinq spectres par dépôt).

Les spectres obtenus comportent plusieurs bandes d'absorption dont la position, le nombre et l'intensité renseignent sur la nature et la concentration des groupements fonctionnels $(O H, C=O$, etc...) et structuraux $\left(\mathrm{CH}_{2}, \mathrm{CH}_{3}, \mathrm{C}=\mathrm{C}\right.$ etc...).

\section{Calcul des indices spectrométriques}

L'épaisseur du dépôt n'étant pas toujours identique d'une mesure à l'autre, pour un même échantillon, l'intensité des différents spectres peut varier. Afin de pouvoir comparer les différents spectres, des indices spectrométriques sont utilisés. Ces indices IRTF sont construits à partir de rapport d'aires de bandes, ce qui permet de quantifier de façon relative les modifications structurales qui se produisent lors du vieillissement. Les rapports d'aires de bande permettent le calcul de plusieurs indices fonctionnels et structuraux [9-11]. A chacun de ces indices correspondent alors des espèces chimiques qui apparaissent ou disparaissent au cours de l'oxydation. Ainsi, ils permettent de suivre l'évolution du vieillissement de chaque émulsion globalement ou espèce par espèce.

\section{Résultats}

Pour tous les échantillons stockés à $25^{\circ} \mathrm{C}$, aucune modification spectrale n'a été observée au cours du temps. Par contre, pour les échantillons stockés à $50^{\circ} \mathrm{C}$, des modifications sont mesurées au niveau des indices relatifs aux bandes $\delta_{\mathrm{CH} 2}$ et $\delta_{\mathrm{c}_{3}}$ (entre 1300 et $1500 \mathrm{~cm}^{-1}$ ) et au niveau de l'indice carbonyle (entre 1680 et $1780 \mathrm{~cm}^{-1}$ ) avec notamment un élargissement de la bande correspondante (figure 1). En effet, ces bandes d'absorption correspondent aux espèces chimiques les plus sensibles aux phénomènes d'oxydation [12-13]. Pour suivre l'évolution du vieillissement des émulsions, ce sont donc ces deux zones spectrales qui ont été principalement étudiées.

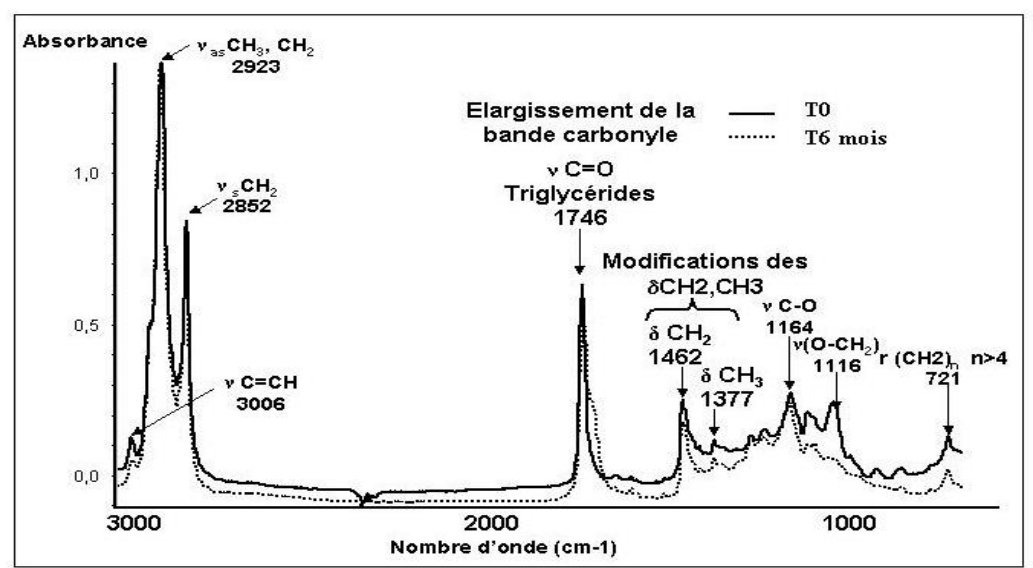

Figure 1 : Spectre IRTF de l'émulsion NAE2 avant et après un vieillissement de 6 mois à $50^{\circ} \mathrm{C}$. 
Afin de pouvoir expliquer les changements apparus au cours du vieillissement des émulsions, les spectres ont été déconvolués. La déconvolution est un traitement mathématique du spectre permettant d'améliorer la résolution spectrale. Elle permet d'affiner le spectre et de mettre en évidence les différentes composantes d'un massif. Les indices utilisés pour décrire les espèces chimiques apparues ou disparues au cours de l'oxydation ont été définis à partir des bandes présentes sur les spectres déconvolués. Ainsi le massif de la bande carbonyle entre $1680 \mathrm{~cm}^{-1}$ et $1780 \mathrm{~cm}^{-1}$ a pu être affiné faisant apparaître différentes bandes spectrales dont les indices sont définis de la façon suivante :

Indices carbonyles (relatif à l'absorption de la bande $v C=O$ ) :

$A_{\lambda} / \Sigma A_{C=0} \quad$ avec $\Sigma \square A_{C=0}=A_{1754}+A_{1746}+A_{1735}+A_{1727}+A_{1719}+A_{1709}+A_{1701}$.

Les modifications des bandes d'absorption entre 1680 et $1780 \mathrm{~cm}^{-1}$ ont été suivies avec les indices suivants :

Indices d'aliphaticité (relatif à l'absorption des bandes $\delta \mathrm{CH}_{2}$ et $\delta \mathrm{CH}_{3}$ ) :

$\mathrm{A}_{\lambda} / \Sigma \mathrm{A}_{\mathrm{CH} 2, \mathrm{CH} 3}$ avec $\Sigma \square \mathrm{A}_{\mathrm{CH} 2, \mathrm{CH} 3}=\mathrm{A}_{1467}+\mathrm{A}_{1458}+\mathrm{A}_{1432}+\mathrm{A}_{1417}+\mathrm{A}_{1401}+\mathrm{A}_{1377}$.

Pour suivre l'évolution de la stabilité des émulsions vis-à-vis de l'oxydation, l'évolution des différents indices en fonction du temps de stockage a été évaluée.

La déconvolution a permis d'une part d'identifier les nouvelles espèces formées au cours du phénomène de vieillissement (cétones, aldéhydes, acides gras libres...) [14] et d'autre part de mettre en évidence la disparition de la liaison $\delta \mathrm{CH}_{2}-\mathrm{O}-\mathrm{CO}$ due à la rupture de triglycérides (figure 2).

Par conséquent, cette étude IRTF met en évidence certains mécanismes d'oxydation. Les nouvelles espèces chimiques qui apparaissent suggèrent une autooxydation des triglycérides (contenus dans la phase grasse de l'émulsion) en hydropéroxydes. En effet, la décomposition des hydropéroxydes entraîne l'apparition d'aldéhydes, de cétones et d'acides gras libres... [15, 16]. De plus, il apparaît également qu'une hydrolyse des triglycérides se produit en parallèle de l'autooxydation lors du vieillissement (disparition de la liaison $\delta \mathrm{CH}_{2}-\mathrm{O}-\mathrm{CO}$ caractéristique des triglycérides).

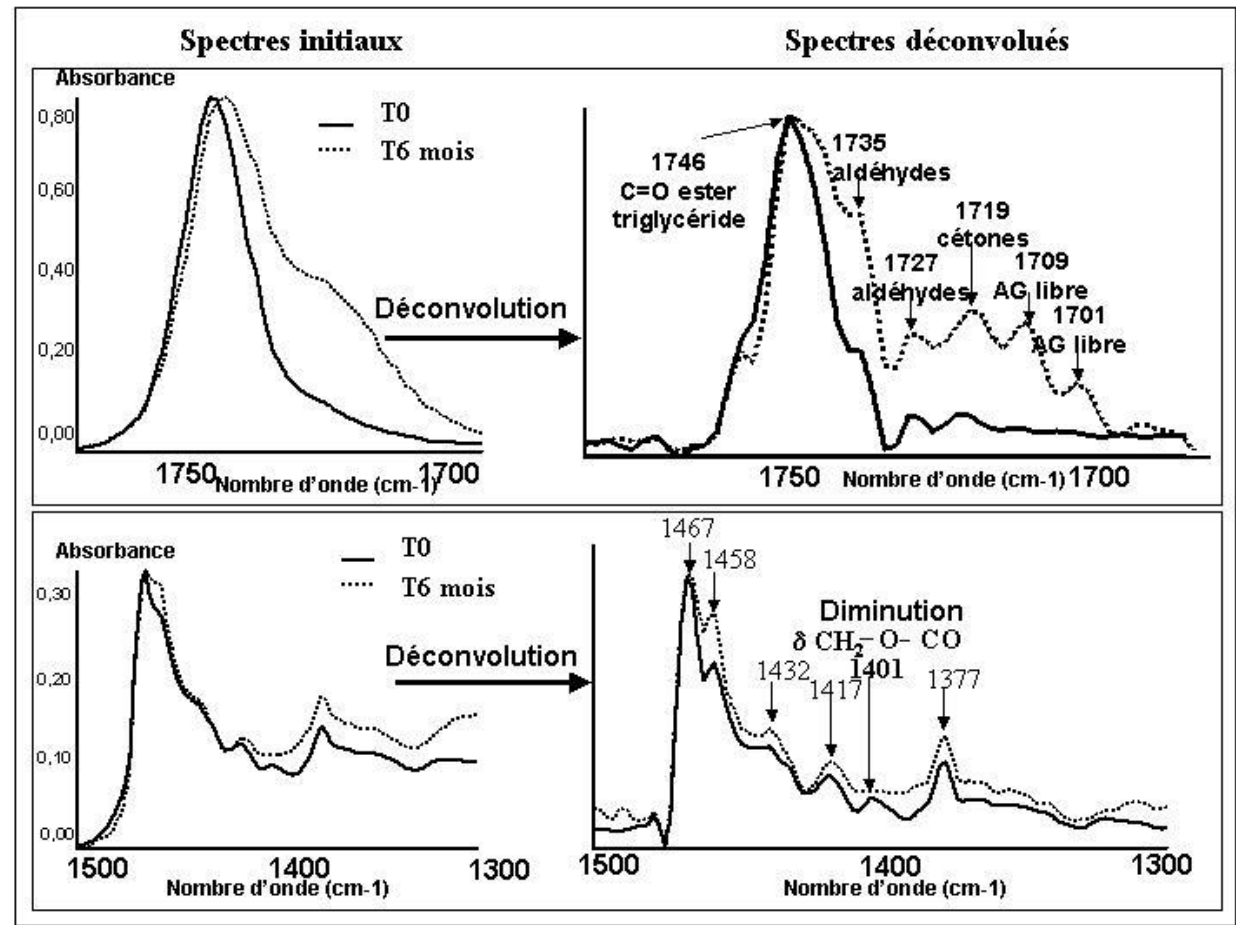

Figure 2 : Déconvolution des spectres avant et après vieillissement de 6 mois à $50^{\circ} \mathrm{C}$.

Les cinétiques d'oxydation de ces espèces ont permis de comparer et de classer les émulsions en fonction de leur stabilité. Le tableau 2 montre une diminution des bandes relatives aux triglycérides et une augmentation des bandes relatives aux espèces issues de l'oxydation (aldéhydes, cétones et acides gras libres). II apparaît clairement que les variations les plus importantes sont obtenues pour 
l'émulsion NAE2 à base d'huile de noyaux d'abricot et de l'émulsifiant E2. En revanche, l'émulsion OE1 semble être la moins sensible à l'oxydation. A partir de ces variations, les émulsions ont pu être classées de la moins stable à la plus stable de la manière suivante :

$$
\text { NAE2 }<\text { OE2 }<\text { NAE1 < OE1 }
$$

II est donc possible grâce à cette technique IRTF d'une part de déterminer quelle sera l'émulsion la plus sensible à l'oxydation et d'autre part, d'identifier les mécanismes mis en jeu lors de cette oxydation. II sera alors possible d'intervenir sur ces mécanismes par l'utilisation d'additifs antioxydants spécifiques.

Tableau 2 : Variations des indices relatifs aux bandes carbonyles déconvoluées entre T0 et T6mois à $50^{\circ} \mathrm{C}$.

Longueurs d'ondes correspondant aux bandes carbonyles déconvoluées $\left(\mathrm{cm}^{-1}\right)$

1754

1746

1735

1727

1719

1709

1701

Triglycérides Triglycérides Aldéhydes Aldéhydes Cétones AG libres AG libres

\begin{tabular}{clllllll}
\hline NAE1 & -40 & -22 & +43 & +98 & +111 & +129 & +154 \\
NAE2 & $-\mathbf{5 4}$ & $-\mathbf{3 7}$ & $\mathbf{+ 5 9}$ & $\mathbf{+ 2 1 0}$ & $\mathbf{+ 2 1 7}$ & $\mathbf{+ 4 1 2}$ & $\mathbf{+ 3 6 9}$ \\
OE1 & $\mathbf{- 2 8}$ & $-\mathbf{1 6}$ & $\mathbf{+ 3 6}$ & $\mathbf{+ 8 3}$ & $\mathbf{+ 9 0}$ & $\mathbf{+ 1 0 7}$ & $\mathbf{+ 1 2 3}$ \\
OE2 & -8 & -23 & +54 & +161 & +141 & +176 & +150 \\
\hline
\end{tabular}

\section{La rhéologie}

Les mesures rhéologiques en régime permanent ou dynamique fournissent des informations sur la stabilité physique de l'émulsion. C'est pourquoi il est essentiel de réaliser des mesures rhéologiques en fonction du temps pour obtenir une information sur la stabilité et la consistance du produit [17]. L'objectif est de pouvoir prédire la stabilité d'une émulsion à long terme à l'aide de mesures rhéologiques réalisées à court terme.

Cette étude en rhéologie est réalisée en parallèle des mesures en spectrométrie IRTF sur les émulsions stockées à $25^{\circ} \mathrm{C}$ et $50^{\circ} \mathrm{C}$.

L'évaluation rhéologique est réalisée à température ambiante $\left(25^{\circ} \mathrm{C}\right)$ suivant les trois procédures suivantes:

- $\quad$ en régime permanent, un test en écoulement $(E C)$

$$
0,2<\tau<120 \mathrm{~Pa} \text {, }
$$

- en régime dynamique, un test en balayage contrainte (SS)

$$
0,01<\tau<200 \mathrm{~Pa} \text { avec une fréquence } \mathrm{F}=1 \mathrm{~Hz},
$$

- $\quad$ en régime dynamique, un test en balayage fréquence (FS)

$$
0,1<\mathrm{F}<30 \mathrm{~Hz} \text { avec une contrainte } \tau=1 \mathrm{~Pa} \text {. }
$$

Ces procédures ont été choisies de manière à pouvoir être appliquer à différents types d'émulsions couvrant une large gamme de viscosité. Ces émulsions sont donc testées à la même température $\left(25^{\circ} \mathrm{C}\right)$, avec le même appareillage (rhéomètre AR1000, TA instrument) et le même mobile (géométrie : cône-plan, diamètre $=6 \mathrm{~cm}$, angle $\alpha=1^{\circ} 59$, entrefer $=68 \mu \mathrm{m}$ ).

Tous ces tests permettent alors d'évaluer les modifications physiques pouvant se produire lors du vieillissement des émulsions dans le temps. L'évolution des crèmes cosmétiques a été suivi à l'aide des paramètres rhéologiques suivants : la viscosité $\eta_{0}$ et le seuil d'écoulement $\tau_{0}$ (test en écoulement), les modules élastique et visqueux G' et G', la tangente $\delta$ et le seuil d'écoulement $\tau_{0}$ (tests en balayage contrainte et balayage fréquence). 
Afin d'assurer une certaine répétabilité, trois mesures sont réalisées pour chaque test sur les deux lots de chaque type d'échantillon. De plus, certains paramètres peuvent être mesurés de plusieurs manières grâce aux différents tests réalisés. En effet, le test en balayage fréquence permet de confirmer les valeurs obtenues en balayage contrainte : les valeurs de G', G' et tangente $\delta$ en balayage contrainte (SS) mesurées dans le domaine de viscoélasticité linéaire (DVEL) doivent se retrouver en balayage fréquence $(F S)$ à $1 \mathrm{~Hz}$. De même, le seuil d'écoulement $\tau_{0}$ peut être mesuré en écoulement, en balayage contrainte et en balayage fréquence.

\section{Résultats}

\section{Influence au stockage}

Les résultats concernant les émulsions stockées à $50^{\circ} \mathrm{C}$ mettent en évidence une augmentation importante de tous les paramètres rhéologiques mesurés jusqu'au déphasage ou crémage de l'émulsion. Lors de la déstabilisation de l'émulsion, il n'est plus possible de prendre en compte les mesures puisqu'elles ne sont plus répétables. En effet, l'émulsion n'étant plus homogène, les trois mesures diffèrent en fonction du prélèvement effectué. C'est pourquoi les mesures ne sont réalisées que jusqu'à 3 ou 4 mois selon la stabilité de l'émulsion.

En ce qui concerne, les émulsions stockées à $25^{\circ} \mathrm{C}$, une diminution de tous les paramètres, exceptée la tangente $\delta$, est mesurée lors du stockage pendant 6 mois. Lorsque l'on compare les mesures réalisées en balayage contrainte, par exemple, entre T0 et T6mois pour une même émulsion (figure 3), une diminution des modules et du seuil d'écoulement apparaissent clairement.

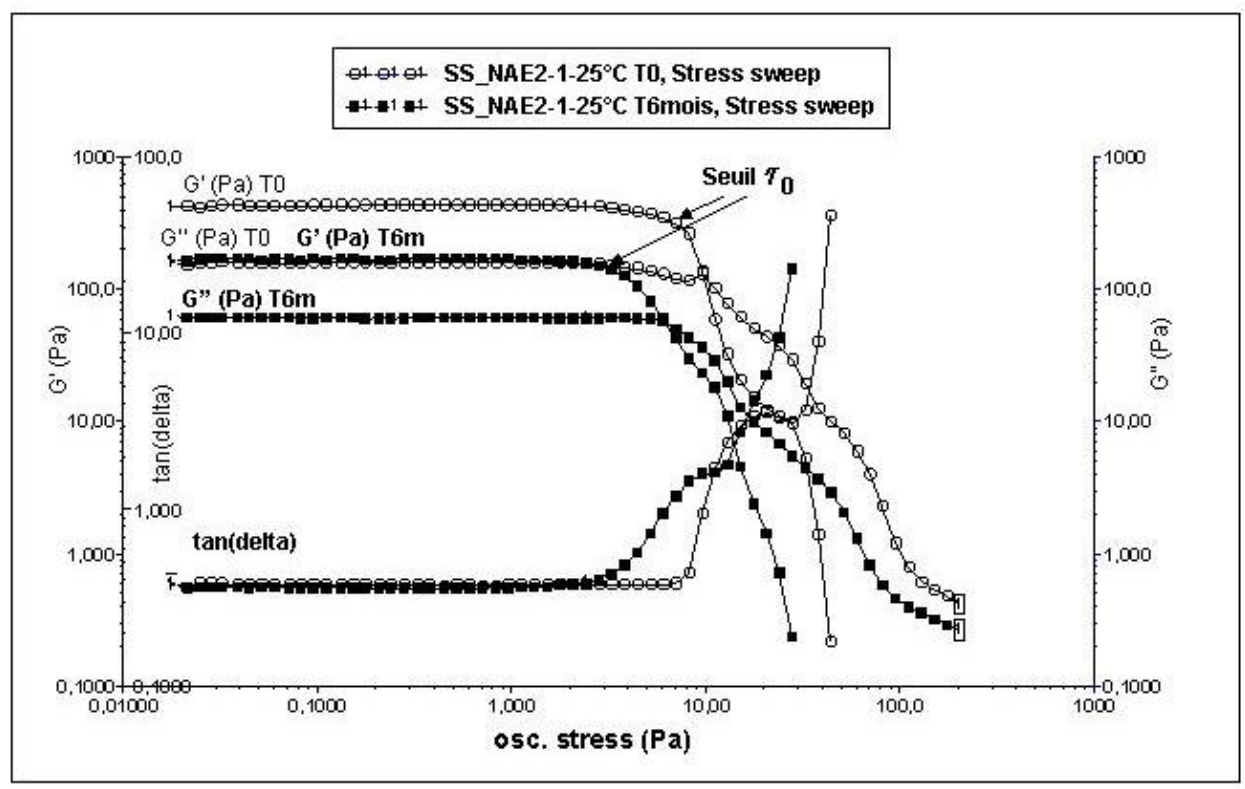

Figure 3 : Comparaison des courbes en balayage contrainte entre T0 et T6mois de l'émulsion NAE2.

Le tableau 3 représente les variations des paramètres rhéologiques mesurés entre le temps T0 et T6mois. De même qu'en spectrométrie IRTF, les variations les plus importantes sont obtenues pour l'émulsion NAE2 à base d'huile de noyaux d'abricot et d'émulsifiant E2. L'émulsion ayant les variations les moins importantes est également OE1 (huile d'olive et émulsifiant E1).

Tableau 3 : Variations des paramètres rhéologiques entre T0 et T6mois.

\begin{tabular}{|c|c|c|c|c|c|c|}
\hline Nom & n0 (EC) & $\tau 0$ (EC) & $\tau 0$ (SS) & G' (SS) & G' (SS) & $\tan \delta(S S)$ \\
\hline NAE1 & $-37 \%$ & $-30 \%$ & $-26 \%$ & $-36 \%$ & $-41 \%$ & $-7 \%$ \\
\hline NAE2 & $-67 \%$ & $-64 \%$ & $-52 \%$ & $-58 \%$ & $-60 \%$ & $-6 \%$ \\
\hline OE1 & $-30 \%$ & $-10 \%$ & $-20 \%$ & $-36 \%$ & $-38 \%$ & $-3 \%$ \\
\hline OE2 & $-55 \%$ & $-45 \%$ & $-33 \%$ & $-57 \%$ & $-57 \%$ & $-1 \%$ \\
\hline
\end{tabular}


Une classification de la stabilité des émulsions a pu également être établie à partir des variations de ces paramètres. D'où le classement suivant, de l'émulsion la moins stable à la plus stable :

$$
\text { NAE2 }<\text { OE2 < NAE1 < OE1 }
$$

II apparaît que l'émulsifiant E2 influe fortement sur la stabilité de l'émulsion puisque les variations les plus importantes sont observées lorsqu'il est présent dans la formulation.

Au second plan, il apparaît également que la nature de l'huile joue également un rôle sur la stabilité de l'émulsion. En effet, les émulsions à base d'huile de noyaux d'abricot sont toujours plus instables que les émulsions à base d'huile d'olive pour un même émulsifiant.

\section{Influence de l'émulsifiant}

Cette étude met également en évidence l'influence de l'émulsifiant sur la valeur des paramètres rhéologiques. En effet, des paramètres ayant le même ordre de grandeur en viscosité, au niveau des modules G' et G" et du seuil d'écoulement sont mesurés pour les émulsions formulées avec le même émulsifiant.

Par exemple, la figure 4 montre les variations du module élastique G' (balayage contrainte) en fonction du temps pour les différentes émulsions étudiées (les deux lots sont représentés pour chacune des émulsions). II apparaît que les émulsions à base d'émulsifiant E2 ont un module élastique G' entre 400 et $500 \mathrm{~Pa}$ alors que pour les émulsions à base d'émulsifiant $\mathrm{E} 1, \mathrm{G}$ ' est beaucoup plus important $\left(\mathrm{G}^{\prime} \approx 1000 \mathrm{~Pa}\right)$. Les émulsions à base d'émulsifiant $\mathrm{E} 1$ ont également une viscosité $\eta_{0}$ et un seuil d'écoulement $\tau_{0}$ plus importants.

Par conséquent, les émulsions les plus stables sont les émulsions ayant une viscosité, un module élastique et un seuil d'écoulement plus importants.

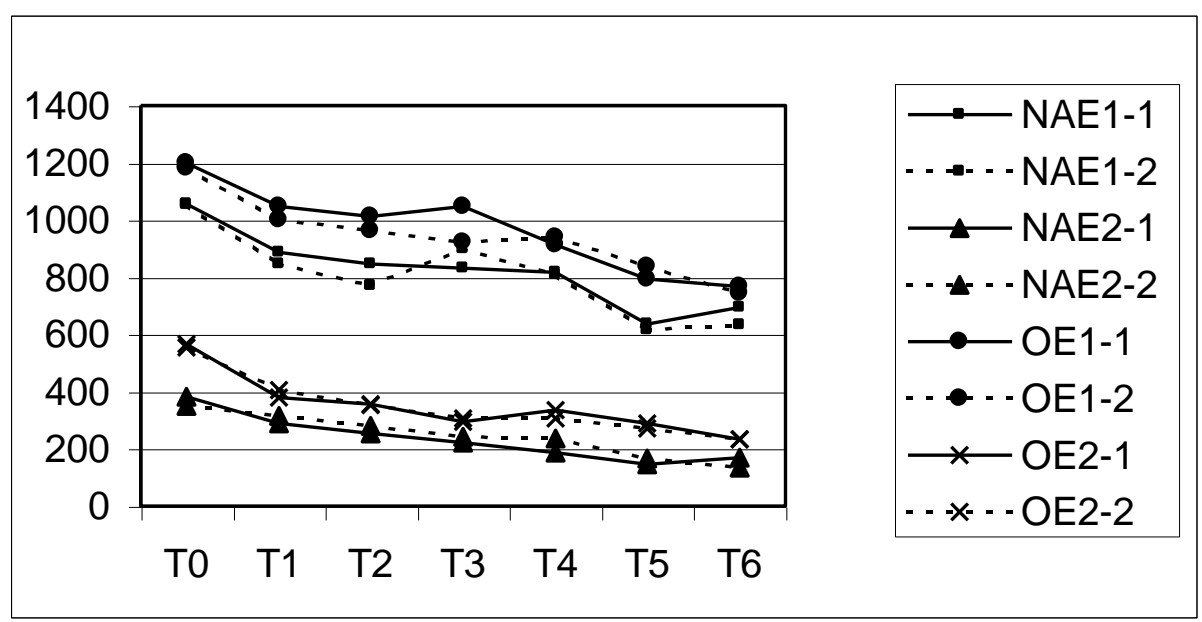

Figure 4 : Evolution du module élastique $G$ ' en fonction du temps pour les 4 types d'émulsion stockées à $25^{\circ} \mathrm{C}$.

\section{La microscopie}

Les mesures de microscopie optique en contraste de phase viennent compléter l'étude rhéologique et spectroscopique en fournissant une interprétation du processus de vieillissement de ces émulsions. Cette technique permet l'étude de la distribution des gouttelettes d'huile au sein de l'émulsion (nombre et taille des gouttelettes) ainsi que la visualisation de leur répartition.

A $25^{\circ} \mathrm{C}$, aucune modification de la taille des globules n'a été mesurée quelque soit l'émulsion étudiée. Par contre à $50^{\circ} \mathrm{C}$, les clichés en microscopie pris tout au long du stockage (vieillissement) permettent la mise en évidence des processus de déstabilisation : 
- les émulsions à base d'émulsifiant E1 montrent directement une coalescence au temps T3mois caractérisée notamment par une augmentation du diamètre des globules et par une diminution de leur nombre (figures 5 a et $5 \mathbf{c}$ ) ;

- les émulsions à base d'émulsifiant E2 révèlent un crémage au temps $\mathrm{T} 1$ et $\mathrm{T} 2 \mathrm{mois}$ avec une augmentation du nombre de globules (figures $\mathbf{5 d}$ et $\mathbf{5 e}$ ), suivi d'une coalescence dès le temps T3mois (figure $5 \mathbf{f}$ ).
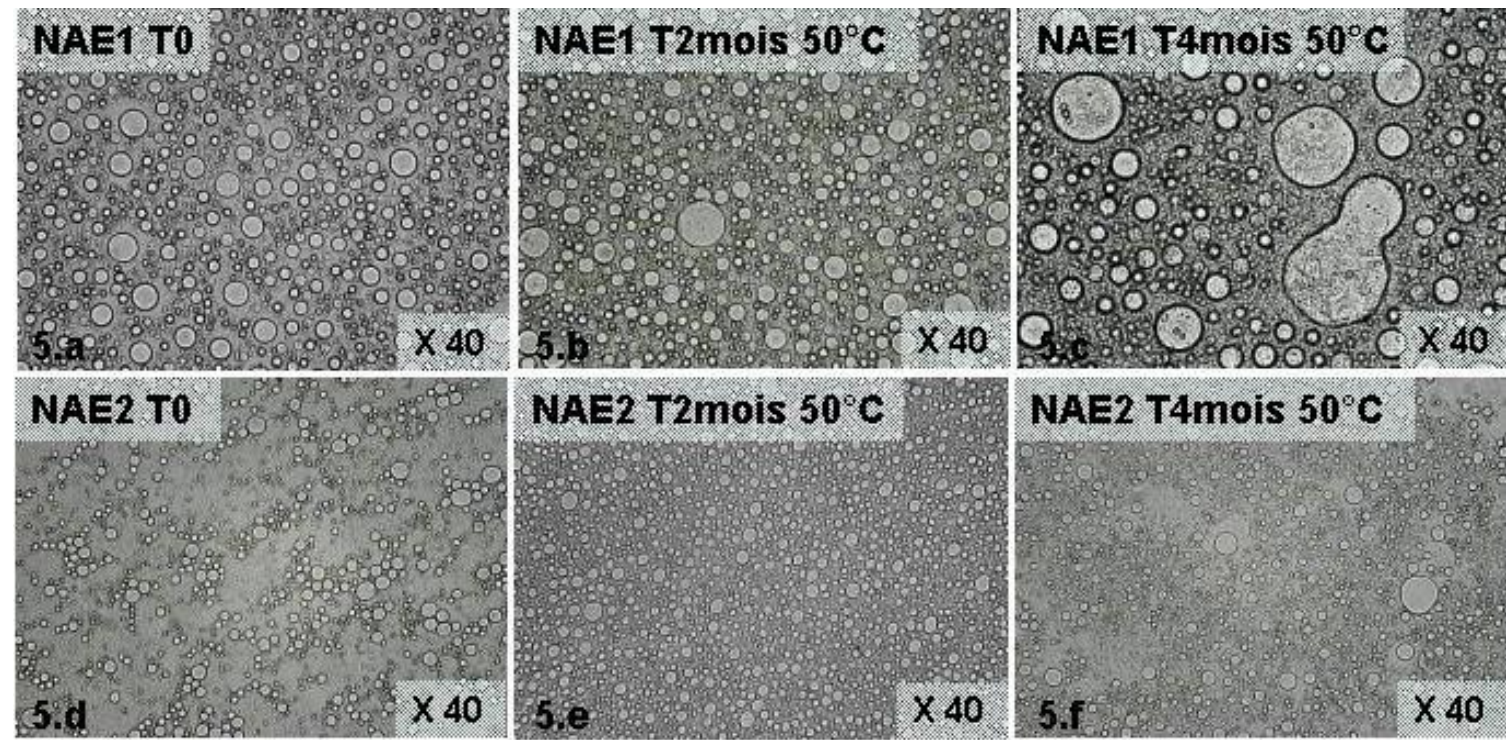

Figure 5 : Evolution des globules par microscopie lors d'un vieillissement accéléré à $50^{\circ} \mathrm{C}$.

\section{Conclusion}

Les résultats obtenus en spectrométrie IRTF $\left(50^{\circ} \mathrm{C}\right)$ et en rhéologie $\left(25^{\circ} \mathrm{C}\right)$ ont permis d'établir une classification des émulsions en fonction de leur stabilité. Ces deux techniques, bien qu'étant complètement différentes (l'une mesurant les modifications chimiques et l'autre les modifications physiques) conduisent au même classement.

L'IRTF et la rhéologie sont donc deux techniques complémentaires. La spectrométrie IRTF a permis d'identifier les modifications structurales au sein des émulsions lors du vieillissement ce qui permet d'avoir une meilleure compréhension des mécanismes d'oxydation. Grâce à cette technique, il est possible d'identifier les produits de dégradation des émulsions et de prédire si une formulation sera sensible à l'oxydation.

Quant à la rhéologie, elle apporte un aspect prédictif puisqu'à $25^{\circ} \mathrm{C}$, des modifications des paramètres rhéologiques sont mesurées alors qu'aucune modification n'est observable ni à l'œil nu, ni en microscopie, ni même en IRTF.

Ces deux techniques complémentaires représentent ainsi un outil important pour la formulation en cosmétologie. Utilisées conjointement, elles permettront de prédire la stabilité d'une émulsion, d'identifier les produits de dégradation issus du vieillissement et de faciliter le choix d'additifs. Ces approches complémentaires peuvent être utilisées pour la formulation d'autres types d'émulsions (agroalimentaire, pétrolier...). 


\section{Références}

[1] A.T. Florence and F. Rieg, In: F. Puissieux and M. Seiller (Eds.), Agents de surface et émulsions : Les systèmes dispersés I, Lavoisier, Tec \& Doc (Galenica 5), 5 Paris (1983) 321-342.

[2] R.P Borwankar; L.A Lobo and D.T. Wasan, Colloids and surfaces. 69 (1992) 135-146.

[3] E. Dickinson, Les colloïdes alimentaires, Masson, Paris (1992) 85-121.

[4] P.C. Hiemenz and R. Rajagopalan, Principles of colloid and surface chemistry, $3^{\text {rd }}$ Ed., Dekker, New York (1997).

[5] R. Barreiro-Iglesias, C. Alvarez-Lorenzo and A. Concheiro, J. Therm. Anal. Calorim. 28 (2002) 479488.

[6] E.L. Sliwinski, P.J. Roubos., F.D. Zoet, M.A.J.S. Van Boekel and J.T.M. Wouters, Colloids Surf. B: Biointerfaces. 31 (2003) 231-242.

[7] M. Gloor, G. Hirsch and U. Willebrandt, Arch. Dermatol. Res. 271 (1981) 305-313.

[8] J. Roussos, In : F. Puissieux and M. Seiller (Eds.), Agents de surface et émulsions : Les systèmes dispersés I, Vol. 5, Lavoisier, Tec \& Doc (Galenica 5), Paris , (1983) pp. 407-421.

[9] M.D. Guillén and N. Cabo, J. Sci. Food Agric. 80 (2000) 2028-2036.

[10] J. Lamontagne, P. Dumas, V. Mouillet and J. Kister, Fuel 80 (2001) 483-488.

[11] A. Permanyer, L. Douifi, A. Lahcini, J. Lamontagne and J. Kister, Fuel 81 (7) (2002) 861-866.

[12] J. Dubois, F.R. Van de Voort, J. Sedman, A.A. Ismail and H.R. Ramaswamy, JAOCS 73 (6) (1996) 787-794.

[13] M. Lazzari, O. Chiantore, Polymer degradation and stability 65 (1999) 303-313.

[14] H. Masmoudi, Y. Le Dréau, P. Piccerelle and J. Kister, International Journal of Pharmaceutics (2005) Sous presse.

[15] A. Karleskind, Manuel des corps gras, Vol. 2, Lavoisier, Tec \& Doc, Paris, (1992).

[16] P.P. Wolf, L'alimentation et la vie, 55 (1967) 295-298.

[17] T. Tadros, Advances in Colloid and Interface Science 108-109 (2004) 227-258. 